\title{
Supervised Method for Face Photo - Sketch Synthesis and Recognition
}

\author{
Shraddha V. Wankhede \\ Electronics and Communication Engineering, \\ Gondwana University, India
}

\author{
Sunil V. Kuntawar \\ Assistant Professor \\ Gondwana University, India
}

\begin{abstract}
Facial Sketches are most widely used in law enforcement agencies for identification and apprehension of suspects, which may involve in several criminal activities. We plan to implement an efficient system to recognize a forensic sketch images to a gallery of mug shot images which will help law enforcement agencies. SIFT algorithm explained for feature extraction. This SIFT feature will possess strong robustness to the accessory, expression, pose, illumination variations. SIFT algorithm have highest percentage of accuracy. Faces are highly deformable objects which may easily change their appearance over time. The feature based sketch matching algorithm (SIFT) we present offers both a simpler and faster design and utilizes localized sketch information in the matching process. SIFT used for extracting local features. Scale invariant feature transform (SIFT) proposed by Lowe has been widely and successfully applied to object detection and recognition. SIFT algorithm's ability has been leveraged through use of MATLAB code. This helps users to use SIFT algorithm very effectively to match sketches with current database images
\end{abstract}

\section{Keywords}

Face sketch recognition, image matching, graphical user interface (GUI), SIFT

\section{INTRODUCTION}

Face Photo -Sketch Recognition \& Synthesis is important technique used to overcome the difficulty of matching photos \& sketches in two different modalities. It is also developed mainly for security purposes thus used in law enforcement. As the necessity for higher levels of security rises, technology is bound to swell to fulfill these needs. Any new creation, enterprise, or development should be uncomplicated and acceptable for end users in order to spread worldwide. To improve discrimination and to provide similar information to face recognition systems, it appears of importance to derive a sketch from a photo when this information is available. This process is called "Face photosketch synthesis".

Face sketch synthesis and recognition is a technology of using computer to analyze the face images and extract the features for recognizing the identity of the target. Due to illumination ,variations and occlusion, the appearances of a face complicate many problems in face recognition. With the rapidly growing number of digital cameras capturing data in public areas, having a robust and accurate face sketch recognition method is critical to apprehend suspects and prevent crimes. Image matching is a research focus in the field of image processing. Usually image in the imaging process will be affected by shooting time, angle ,the natural environment and other factors, not only the image captured by the interference noise and there are serious distortions and abbreviations[6].

The feature based sketch matching algorithm, scale invariant feature transform (SIFT) we present simpler and faster design and utilizes localized sketch information in the matching process. For such useful technique face recognition there are many more algorithms or methods research and develop for the ease of user. Mainly these methods are best favorable on their accuracy. SIFT feature posseses strong robustness to the accessory, expression, pose, illumination variations. SIFT used for extracting local features. The face sketch recognition using SIFT can robustly identify face among clutter and occlusion [9][10].

The Proposed system will accurately matches forensic sketches with their corresponding photo images using feature-based approach. User will feed forensic sketch to the system to find a suitable match for the respective image found in the database. The feature descriptors of both the sketch and photo images are computed and matched for equality, once a match is found the user is alerted and the corresponding suspect's information is extracted from the database and displayed to the user

\section{LITERATURE SURVEY}

Tayyaba Hashmi (2016) states that, The problem of matching a sketch to a gallery of mug shot images is addressed. Previous research in sketch matching only offered solutions to matching highly accurate sketches that were drawn while looking at the subject (viewed sketches). To identify sketches an efficient algorithm is presented here. Both sketches and photos are considered for extracting feature descriptors using SIFT (Scale Invariant Feature Transform). Proposed method can be used to match a few sketches against a mug shot gallery containing several images. The proposed project will lead to state-of-the-art accuracy when matching viewed sketches. The proposed project represent both sketches and photos using SIFT feature descriptors and multiscale local binary patterns (MLBP) [1]. Yukti Bakhshi, Sukhvir Kaur and Prince Verma (2016) states that Face recognition has recently grown its importance, especially during the past several years as one of the most successful applications of image analysis. It has been a fast growing, interesting and challenging area in real time applications. In last decades a large number of face recognition algorithms have been developed. In this paper an attempt is made to review methods used for face recognition- SIFT, SURF, PCA, PCA-SIFT, etc. for recognition and matching. Scale invariant feature transform (SIFT) used to extract distinct invariant features from images can be used to perform reliable matching. We described the basic process of face recognition system and improvement in matching the invariant faces in this paper. We are using SIFT and SURF to extract the features and then applying PCA to the image for the better performance in terms of rotation, pose and illumination. Performance can be seen on the basis of FAR, FRR, Recognition rate and Computation time. For the implementation of this proposed work we use the Image Processing Toolbox under MATLAB Software [2].

Ashwini D.Gadekar and Sheeja S. Suresh (2015) proposed approach, here main focus on the face recognition system. It will one of the new face recognition system based on an 
efficient design. This paper present proposed methodology of global thresholding technique, SIFT (Scale Invariant Feature Transform), PCA (Principal Component Analysis) and SVM (Support Vector Machine) classifier. SIFT is used for extract the feature from faces. This SIFT feature will possess strong robustness to the accessory, expression, pose, illumination variations. PCA is a standard technique use for dimensionality reduction in which face data can analyze and observation can be described by several inter-correlated dependent variables[3].

Poonam A. Katre (2014) states that faces are highly deformable objects which may easily change their appearance over time. Not all faces areas are subject to the same variability. The SIFT approach in the context of face authentication. Several pattern recognition and classification techniques have been applied to the biometrics domain. The SIFT (scale invariant feature transform) is used for extracting local features [5]

.Xue Leng and Jinhua Yang (2014) presents that Image matching is a research focus in the field of image processing. The method based on feature matching by researchers alike for its stable performance. Based on the analysis of the traditional SIFT algorithm, for computationally intensive problems of SIFT feature descriptor links, Proposed a new descriptor generation algorithm. Experimental results show that this method can effectively reduce the amount of calculation, improve processing speed [6].

Amit R. Sharma and Prakash R. Devale (2012) states that face Recognition has gained much attention in the field of network multimedia access. After the 9/11 tragedy in India, the need for technologies for identification, detection and recognition of suspects has increased. One of the most common biometric recognition techniques is face recognition since face is the convenient way used by the people to identify each other. In this paper we are going to study a method for representing face which is based on the features which uses geometric relationship among the facial features like mouth, nose and eyes .Feature based face representation is done by independently matching templates of three facial regions i.e. eyes, mouth and nose[8] .

Andrea Vedaldi (2007) describes an implementation of the Scale-Invariant Feature Transform (SIFT) detector and descriptor. The implementation, which is compatible with D. Lowe's implementation1, is distributed along with the source code. Designed for the MATLAB environment, the code is broken into several $\mathrm{M}$ and MEX files that enable running selected portions of the algorithm [11].

David G. Lowe (2004) present a method for extracting distinctive invariant features from images that can be used to perform reliable matching between different views of an object or scene. The features are invariant to image scale and rotation, and are shown to provide robust matching across a substantial range of affine distortion, change in $3 \mathrm{D}$ viewpoint, addition of noise, and change in illumination [12].

YU MENG and Dr. Bernard Tiddeman (supervisor) states that The SIFT algorithm takes an image and transforms it into a collection of local feature vectors. Each of these feature vectors is supposed to be distinctive and invariant to any scaling, rotation or translation of the image. In the original implementation, these features can be used to find distinctive objects in different images and the transform can be extended to match faces in images. This report describes our own implementation of the SIFT algorithm and highlights potential direction for future research [13].

\section{PROPOSED METHODOLOGY}

In 2004 Lowe, invents SIFT descriptor which is invariant to scale, rotation, affine transformation, noise, occlusions and is highly distinctive. SIFT features consist of four major stages in detection and representation; they are (1) finding scale-space extrema; (2) key point localization and filtering; (3) orientation assignment; (4) key point descriptor. The first stage is to construct the key points of images by using Difference-ofGaussian (DoG) function. The second stage, candidate key points are restricted to sub-pixel accuracy and removed if found to be unreliable. The third stage represents the dominant orientations for each essential point of the images. The final stage constructs a descriptor for each key point location depends upon the image gradients in its local neighborhood. Then the SIFT descriptor is accepting the 128- dimensional vector which used to identify the neighborhood around a pixel. The SIFT extracts the key points (locations and descriptors) for all the database images. Then given an altered image SIFT extracts the key point for that image and compares that point to the database [2].

\section{FLOW DIAGRAM OF SIFT ALGORITHM}

Face detection and tracking is the method to find out determining whether face is present or not in an image. Face detection gives information about presence or absence of required face only which is contrary to the face recognition technique. The group of feature based face recognition techniques is the Scale Invariant Feature Transform (SIFT) proposed by Lowe. The SIFT technique and its corresponding features has many properties that make them suitable for matching different images of an object or a scene. The SIFT is a method that detects the local key points that are notable and stable for images in different resolutions and uses scale and rotation invariant descriptors to represent the key-points .Face recognition is done by using SIFT algorithm. The input query image first extracted the key points. If the search input image key points are close to train database than the input query image is matches with the database [2].

A SIFT feature is a selected image region like local feature eyes, nose and mouth (also called keypoint) with an associated descriptor. Keypoints are extracted by the SIFT detector and their descriptors are computed by SIFT descriptor. The scale invariant feature transform (SIFT) is an algorithm in computer vision to detect and describe local features in images. (see Figure 1)

\section{RESULT AND ANALYSIS 5.1 Collection of Face Database}

Following figures is collection of face database utilize for face sketch recognition using MATLAB GUI. Keypoints are match within this database figures and whatever keypoints matched are shown in results and corresponding highest keypoint matching figures get selected.(see Figure 2)This image represent first screen of GUI, this is the training GUI we have utilize for results and analysis purpose. The sketch on the right hand side of the image is the sketch utilized for keypoint matching purpose. (see Figure 3)

The figure 4 shows the second screen of MATLAB GUI after we click search button. When we click search button MATLAB run SIFT algorithm code and extracting features and match keypoints.The process results in images shown left hand side of the figure. The different number of keypoints matched within database. On the basis of number of keypoint match images are arranged in the sequence of respective approximation. This 
means that first approximation image is selected because the highest numbers of selected sketch are matched with it and respectively for other approximations.(see Figure 4)

The image above is the first approximation with highest number of keypoint match that is 50 keypoints. Input sketch based on trained photograph detect face image having highest keypoint after extracting features that is in above figure of closest image with the sketch image having matching keypoints are more. Face sketch recognition is done by using SIFT algorithm. Input query image first extracted the keypoints. If the search input sketch image keypoints are close to train database than the input query sketch image is matches with the database. The system receives the input face sketch image and recognizes the face from the training set. After completing the recognition process then finding the Euclidean distance between the input face sketch image and training set face, finally shows the result using MATLAB R2013a.One of the images is taken as test image and considers rest as training image. The important features of face are extracted and similarity measure between training image and test image is taken. Finally, the person who receives minimum distance is chosen as the best match. (see Figure 5)

For such useful technique face recognition there are many more algorithms or methods research and develop for the ease of user. Mainly these methods are best favorable on their accuracy.
Alaa Tharwat, Hani Mahdi, Adel El Hennawy and Aboul Ella Hassanien published their work[4] "Face Sketch Recognition Using Local Invariant Features" in 2015 Seventh International Conference of Soft Computing and Pattern Recognition (SoCPaR 2015) and concluded following table(see Table 1)

As shown in table1 SIFT algorithm achieved results better than all other related work that are listed in table which used the same database. So we have selected to leverage SIFT method using MATLAB software for end users.

\section{CONCLUSION}

Face sketch recognition is a challenging as well as important recognition technique. There is a need for continual research on forensic sketch matching. This can help assist the law enforcement agencies to apprehend criminals quickly, before they commit another crime. In this paper SIFT algorithm utilizes feature extraction and keypoint matching process for the selection of best image by matching highest number of keypoints. Feature matching method by researchers alike for its performance and stability, matching speed. The SIFT algorithm have a good effect for solving image distortion caused by viewpoint change, rotation and scaling and partial occlusion. The advantage of SIFT algorithm posseses strong robustness to the accessory, expression, pose, illumination variation.

Table 1 : Performance of various sketch/photo matching algorithm

\begin{tabular}{|l|l|l|}
\hline \multirow{2}{*}{ Author } & Method & Accuracy (in \%) \\
\hline X. Tang et al. & Eigentransform+PCA & 75 \\
\hline X. Tang et al. & Eigentransform+Bays & 81.3 \\
\hline Qingshan Liu et al. & LDA & 85 \\
\hline Qingshan Liu et al & PCA & 64.33 \\
\hline Xinbo et al. & E-HMM+PCA & 95.24 \\
\hline Xiaoou et al. & PCA & $81.3-97$ \\
\hline Alaa Tharwat & SIFT & 96.25 \\
\hline Alaa Tharwat & LBP & \\
\hline
\end{tabular}




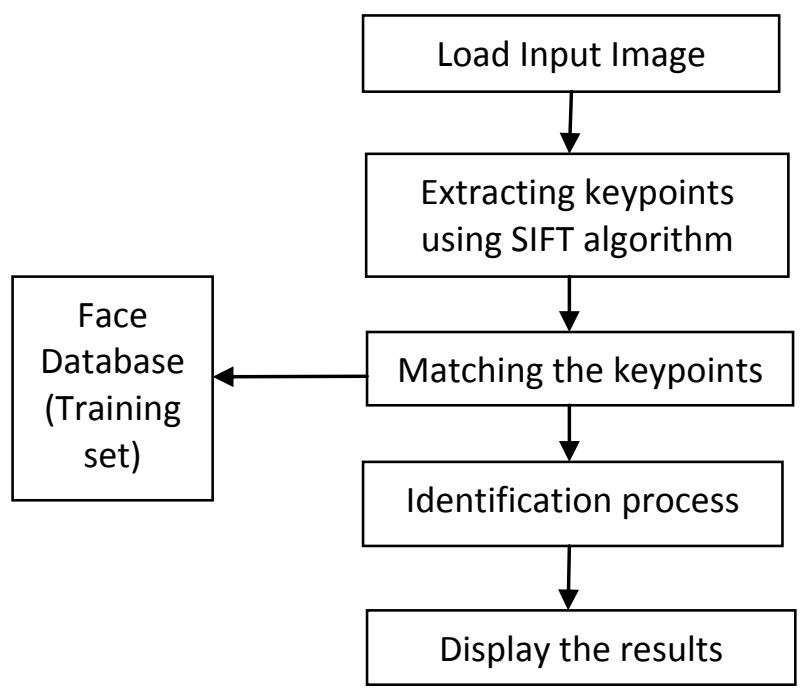

Figure1 : Flow Diagram of SIFT algorithm

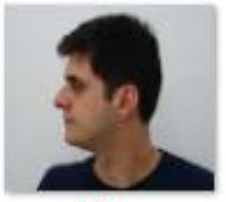

(1).jpg

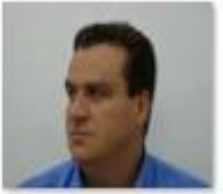

(12).jpg

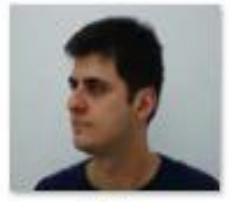

(2).jpg

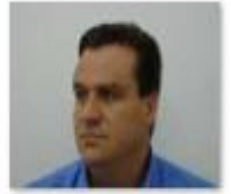

(13).jpg

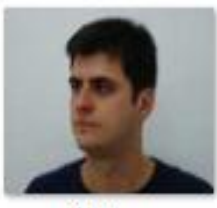

(3).jpg

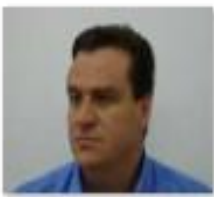

(14).jpg

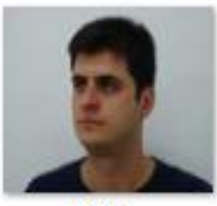

(4).jpg

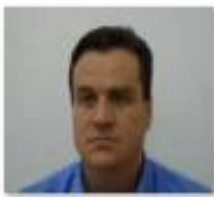

(15).jpg

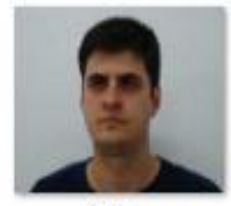

(5).jpg

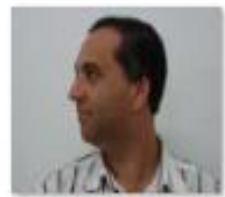

(16).jpg

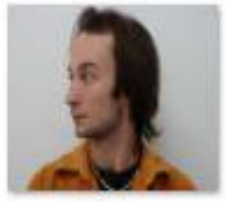

(6).jpg

Figure 2: Collection of Database 
SKETCH TO FACE MATCHING FOR FORENSIC SCIENCE
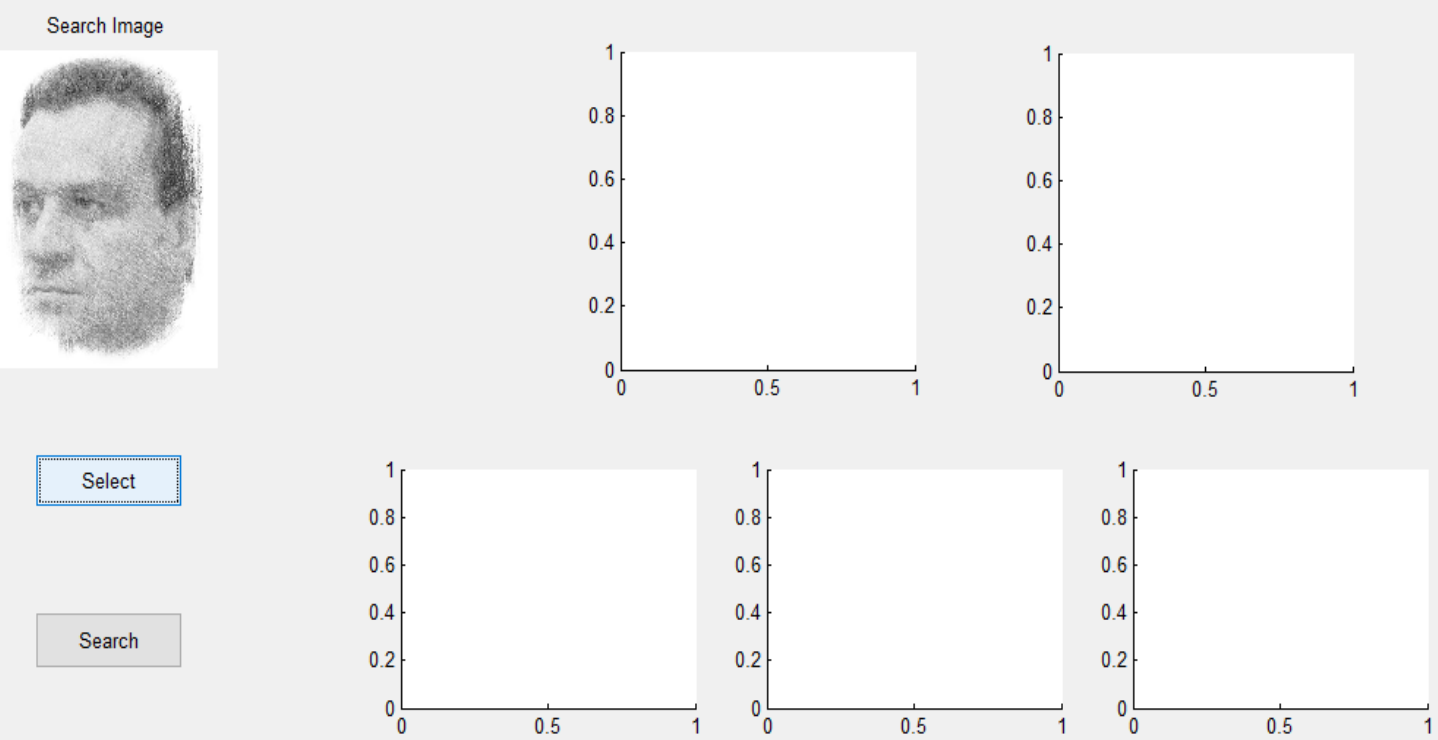

New Training

Click on Search to find face image

Figure 3: The first screen of GUI for search sketch image

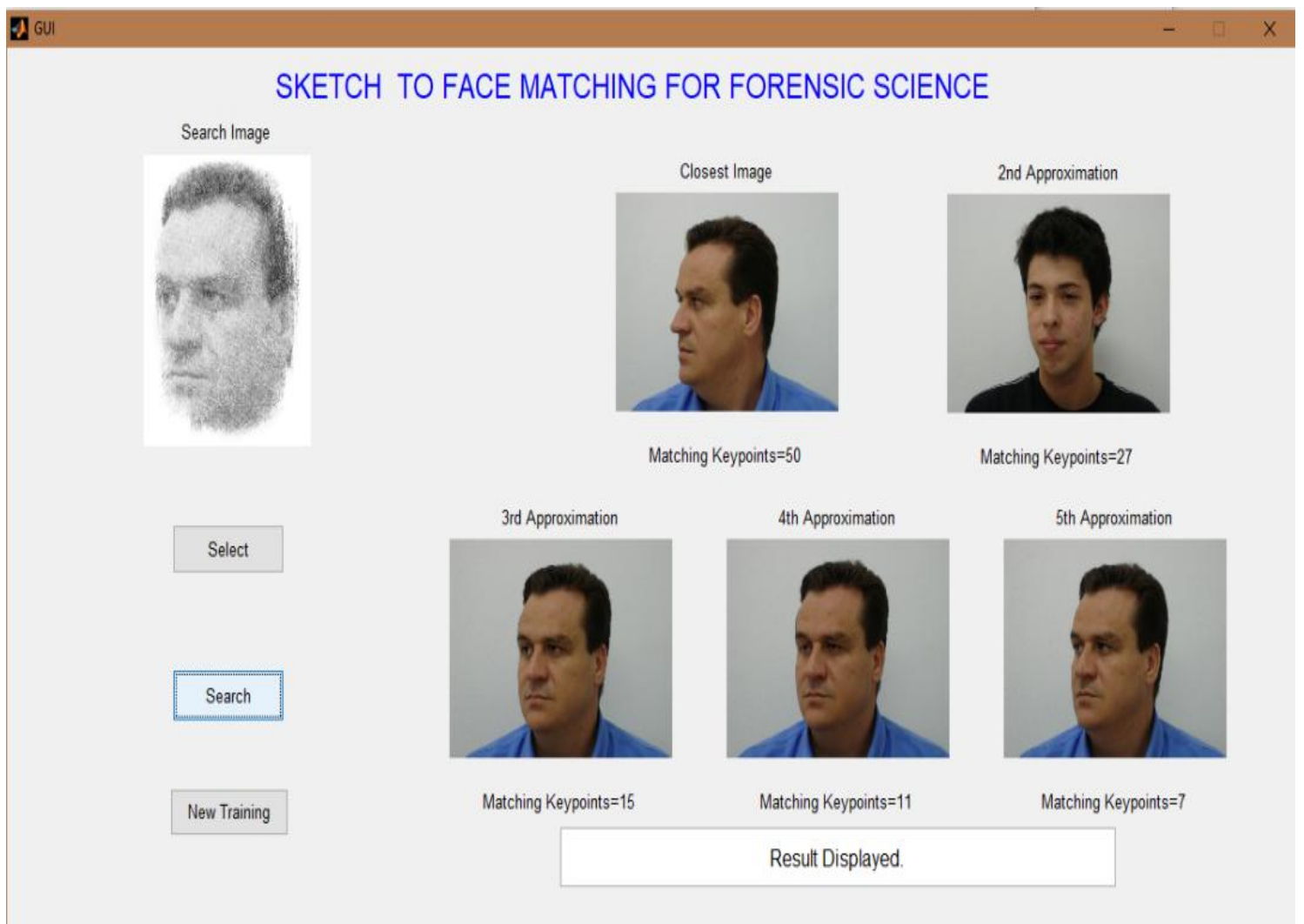

Figure 4: The second screen of GUI showing extracting features from images and matching keypoints with the sketch image. 


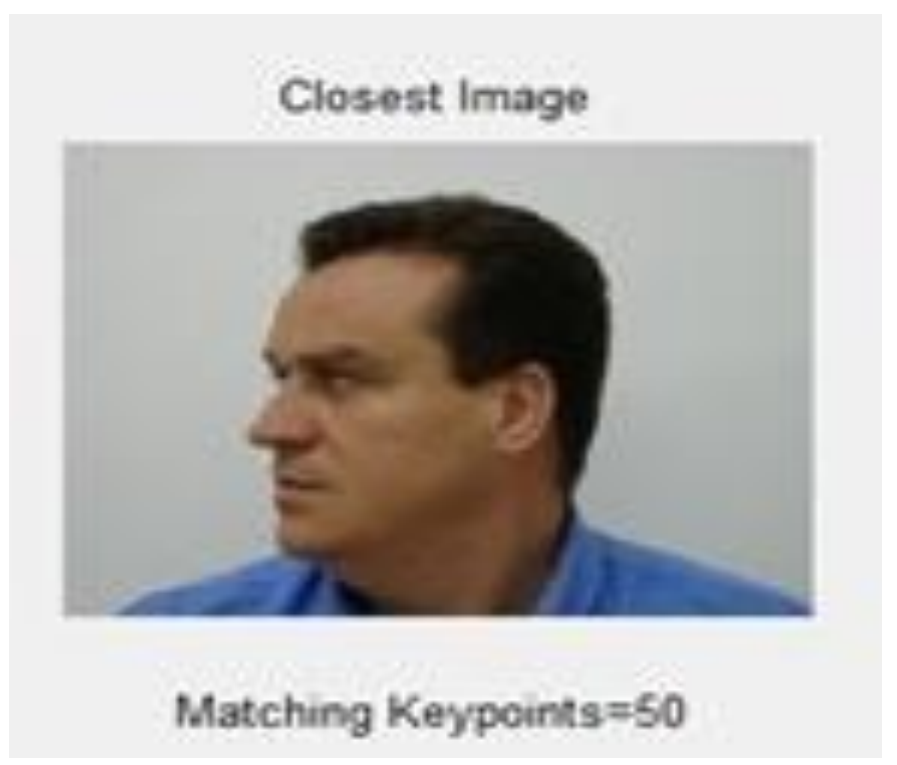

Figure 5: Closest image of matching keypoints are $\mathbf{5 0 .}$

\section{REFERENCES}

[1] Tayyaba Hashmi, "Photograph Retrieval Based On FaceSketch Using Sift With PCA" International Journal of Advance Research In Science And Engineering Volume No.5, Issue No.02, February 2016.

[2]Yukti Bakhshi, Sukhvir Kaur and Prince Verma , "An improvement in face recognition for invariant faces" International journal of current engineering and technology ,Volume 6, No. 2 ,April 2016.

[3]Ashwini D. Gadekar and Sheeja S. Suresh, "Face recognition using SIFT- PCA Feature extraction and SVM classifier" IOSR journal of VLSI and Signal Processing, Volume 5 , Issue 2, April 2015.

[4]Alaa Tharwat, Hani Mahdi, Adel El Hennawy and Aboul Ella Hassanien, "Face Sketch Recognition Using Local Invariant Features" 2015 Seventh International Conference of Soft Computing and Pattern Recognition.

[5] Poonam A. Katre, "Face Authentication /Recognition System For Forensic Application Using Sketch Based On The Sift Features Approach" International Journal of Science, Engineering and Technology Research (IJSETR), Volume 3, Issue 4, April 2014'.

[6]Xue Leng and Jinhua Yang, "Research on improved SIFT algorithm" Journal of Chemical and Pharmaceutical Research, 2014, 6(7):2589-2595.

[7] Riddhi Patel and Shruti B. Yagnik, "A Literature Survey on Face Recognition Techniques" International Journal of Computer Trends and Technology (IJCTT) - volume 5 number 4 -Nov 2013.

[8] Amit R. Sharma and Prakash. R. Devale, "An application to human face photo- sketch synthesis and recognition"
International Journal of Advances in Engineering \& Technology, May 2012.@IJAET Vol. 3, Issue 2, pp. 395402.

[9] B. Klare, Z. Li, and A. K. Jain, "Matching forensic sketches to mug shot photos," IEEE Trans on Pattern Analysis and Machine Intelligence, vol. 33, no. 3 ,2011, pp. 639-646.

[10]Brendan Klare and Anil K Jain, "Sketch to photo matching : A Feature based approach" SPIE conference on Biometric Technology for Human Identification VII, 2010.

[11]Andrea Vedaldi , "An open implementation of the SIFT detector and descriptor" UCLA CSD Technical Report 070012-(2007)

[12] D. Lowe, "Distinctive image features from Scale-Invariant Keypoints," International Journal of Computer Vision, vol. 60(2), pp. 91-110, 2004.

[13]YU MENG and Dr. Bernard Tiddeman(supervisor), "Implementing the Scale Invariant Feature Transform(SIFT) Method".

[14] X.Tang and X. Wang, "Face photo recognition using sketch", in image processing. 2002 proceedings,2002 international conference on vole 1. IEEE 2002, pp , I-257.

[15] R. Uhl and N. Lobo, "A framework for recognizing a facial image from a police sketch, in Proc. Int. Conf. Computer Vision and Pattern Recognition. 1996. Pp. 586593.

[16]M.Turk and A. Petland. "Eigen faces for recognition", Journal of Cognitive Neuroscience. Vol.13, no.1, pp. 7186, 1991.

[17]Yan Ke and Rahul Sukthankar, "PCA-SIFT: A More Distinctive Representation for Local Image Descriptors". 